\title{
MAXIMUM PRINCIPLES FOR PARABOLIC EQUATIONS
}

\author{
GIOVANNI PORRU and SALVATORE SERRA
}

(Received 7 February 1991; revised 12 November 1991)

Communicated by A. J. Pryde

\begin{abstract}
Let $u(x, t)$ be a smooth function in the domain $Q=\Omega \times(0, L], \Omega$ in $\mathbb{R}^{n}$, let $D u$ be the spatial gradient of $u(x, t)$ and let $\nabla u=\left(D u, u_{t}\right)$. If $u(x, t)$ satisfies the parabolic equation $F\left(u, D u, D^{2} u\right)=u_{t}$, we define $w(x, t)$ by $g(w)=|\nabla u|^{-1} G(\nabla u)(g$ is positive and decreasing, $G$ is concave and homogeneous of degree one) and we prove that $w(x, t)$ attains its maximum value on the parabolic boundary of $Q$. If $u(x, t)$ satisfies the equation $\Delta u+2 h\left(q^{2}\right) u_{i} u_{j} u_{i j}=$ $u_{t}\left(q^{2}=|D u|^{2}, 1+2 q^{2} h\left(q^{2}\right)>0\right)$ we prove that $q f(u)$ takes its maximum value on the parabolic boundary of $Q$ provided $f$ satisfies a suitable condition. If $u(x, t)$ satisfies the parabolic equation $a^{i j}(D u) u_{i j}-b(x, t, u, D u)=u_{t}$ (b is concave with respect to $(x, t, u)$ ) we define $C(x, y, t, \tau)=u(z, \theta)-\alpha u(x, t)-\beta u(y, \tau)(0<\alpha, 0<\beta, \alpha+\beta=1, z=\alpha x+\beta y$, $\theta=\alpha t+\beta \tau)$ and we prove that if $C(x, y, t, \tau) \leq 0$ when $x, y, z \in \Omega$ and one of $t, \tau=0$, and when $t, \tau \in(0, L]$, and one of $x, y, z, \in \partial \Omega$, then it is $C(x, y, t, \tau) \leq 0$ everywhere.
\end{abstract}

1991 Mathematics subject classification (Amer. Math. Soc.): 35 B 50.

\section{Introduction}

For elliptic and parabolic equations various maximum principles have been known for a long time [11]. In recent years some maximum principles have been obtained for expressions involving the gradient of solutions. In [12] it is proved that if $u$ is a smooth solution of the elliptic equation $F\left(u, D u, D^{2} u\right)=0$ in a bounded domain $\Omega$ of $\mathbb{R}^{n}$ and $v$ is a fixed direction, then the angle between $v$ and the gradient $D u$ (assumed to be non-vanishing) takes its maximum value on the boundary of $\Omega$. The previous result has been extended to parabolic equations

(C) 1994 Australian Mathematical Society 0263-6115/94 \$A2.00+0.00 
(defined in $\Omega \times[0, L] \subset \mathbb{B}^{n} \times \mathbb{B}$ ) in [13] in case $D u=\left(u_{1}, \ldots, u_{n}\right)$ (the spatial gradient), and in [1] in case $\nabla u=\left(u_{1}, \ldots, u_{n}, u_{t}\right)$. Here $u_{i}(i=1, \ldots, n)$ is the derivative of $u$ with respect to $x_{i}$ and $u_{t}$ is the derivative with respect to $t$, $t \in(0, L]$.

In [10] the elliptic equation $\Delta u+2 h\left(q^{2}\right) u_{i} u_{j} u_{i j}=0$ is investigated. Here $\Delta$ is the Laplace operator, the summation convention (from 1 to $\mathrm{n}$ ) is in effect, $q^{2}=u_{i} u_{i}$ and $h\left(q^{2}\right)$ is a smooth function satisfying $1+2 q^{2} h\left(q^{2}\right)>0$. In [10] it is proved that if $u$ is a smooth solution of this equation in a bounded domain $\Omega$ then the function $q f(u)$ takes its maximum value on the boundary of $\Omega$ provided $f$ satisfies a suitable condition. Similar results were previously obtained in $[7,9,8]$ especially for harmonic and $p$-harmonic functions. (If $h\left(q^{2}\right)=(p-2) / q^{2}$ then the previous equation reads as $\left(q^{p-2} u_{i}\right)_{i}=0$ and its solutions are the usual $p$-harmonic functions.)

In Section 3 of this paper we consider a solution $u$ of the equation $u_{t}=$ $F\left(u, D u, D^{2} u\right)$ and we define a function $w(x, t)$ by $\rho g(w)=G\left(u_{1}, \ldots, u_{n}, u_{t}\right)$, where $\rho=|\nabla u|, g$ is a smooth positive decreasing function and $G$ is a smooth concave function, positively homogeneous of degree one. We prove that $w(x, t)$ assumes its maximum value on $\partial \Omega$. If $v$ is a fixed direction in $\mathbb{R}^{n+1}, G=v \cdot \nabla u$ and $g(w)=\cos (w)$ then we obtain the result of [1]. This maximum principle may be used to investigate the shape of the level sets of $u(x, t)$.

Furthermore we extend the result of [10] to a parabolic equation. Namely, let $u$ satisfy $\Delta u+2 h\left(q^{2}\right) u_{i} u_{j} u_{i j}=u_{t}$, where the left hand side acts on the spatial variables $x_{1}, \ldots, x_{n}$ only and $q=|D u|$. We prove that if $u(x, t)$ is a solution of this equation in $Q=\Omega \times(0, L]$ and $f$ satisfies a suitable condition, then $q f(u)$ takes its maximum value on the parabolic boundary of $Q$. We also prove a maximum principle for $q f(v)$ where $q$ and $f$ are the same as before, whereas $v$ is a solution of an associated parabolic equation. As an application we find a new estimate for the gradient of a solution to the classical heat equation.

In order to investigate the convexity of the solutions of certain parabolic equations, the following concavity function is introduced in [5]:

$$
C(x, y, t)=u(z, t)-\alpha u(x, t)-\beta u(y, t), \quad z=\alpha x+\beta y,
$$

where $x, y, z \in \Omega, t \in(0, L], \alpha$ and $\beta$ are positive real numbers satisfying $\alpha+\beta=1$. If $\Omega$ is convex and $C(x, y, t) \leq 0$ for all $x, y \in \Omega$ and $t \in(0, L]$ then the function $u(x, t)$ is convex with respect to $x$. In [6] it is proved that if $u(x, t)$ is a smooth solution of the parabolic equation

$$
a^{i j}(D u) u_{i j}-b(x, t, u, D u)=u_{t},
$$


where $\partial b / \partial u \geq 0$ and $b$ is jointly concave with respect to $(x, u)$ then, if $C(x, y, t)$ is anywhere positive, its maximum value is attained at some point ( $x, y, t$ ) satisfying: $t=0$ or one of $x, y, z \in \partial \Omega$ (the boundary of $\Omega$ ). Consequently, if $C(x, y, t) \leq 0$ when $x, y, z \in \Omega$, and $t=0$ and when $t \in(0, L]$, and one of $x, y, z \in \partial \Omega$ then necessarily $C(x, y, t) \leq 0$ everywhere.

In Section 4 of the present paper we consider the equation (1.2) where $b$ is jointly concave with respect to $(x, t, u)$, and, for a solution $u(x, t)$ of this equation we define the (more general) concavity function

$$
\begin{aligned}
C(x, y, t, \tau) & =u(z, \theta)-\alpha u(x, t)-\beta u(y, \tau), \\
z & =\alpha x+\beta y, \\
\theta & =\alpha t+\beta \tau .
\end{aligned}
$$

We prove that if $C(x, y, t, \tau) \leq 0$ when $x, y, z \in \Omega$, and one of $t, \tau=0$, and when $t, \tau \in(0, L]$, and one of $x, y, z, \in \partial \Omega$, then necessarily $C(x, y, t, \tau) \leq 0$ everywhere. We remark that no use of the condition $\partial b / \partial u \geq 0$ is made.

We close this introduction with some examples of problems described by the equations involved in the present paper. Equation $u_{t}=F\left(u, D u, D^{2} u\right)$ is a general autonomous evolution equation, and $u_{t}=\Delta u+h\left(q^{2}\right) u_{i} u_{j} u_{i j}$ is a special case of it. When $F\left(u, D u, D^{2} u\right)=K \Delta u$ (K a positive constant) we obtain the classical heat equation for a homogeneous medium in the absence of heat sources [3, p. 41]. If $F\left(u, D u, D^{2} u\right)=\Delta\left(u^{m}\right), m>1$, the corresponding equation describes the flow through a porous media [4, p. 121]. If $F\left(u, D u, D^{2} u\right)=\Delta u+u g\left(u^{2}\right)$, where $\mathrm{g}$ is a suitable smooth function, we find a parabolic dissipative equation [2, p. 26]. Equation (1.2) includes also some non-autonomous equations. This is the case of the heat equation in presence of heat sources [3, p. 41]. The population genetic equation is a special case of (1.2) [3, p. 43]. More examples may be found in [3] and [2].

\section{Notation and preliminary results}

Throughout this paper we denote by $\Omega$ a bounded domain of $\mathbb{R}^{n}$, by $\partial \Omega$ its boundary, by $Q$ the domain $Q=\Omega \times(0, L]$ and by $(x, t)$ a point in $Q$. Subscripts denote partial derivatives; for example, $u_{i}$ is the derivative of $u$ with respect to $x_{i}$. We use the convention that the sum from 1 to $n$ is understood over the repeated indices $i, j$ and $k$. For the indices $r$ and $s$ we use a slightly different convention: $u_{r}$ means the derivative of $u$ with respect to $x_{r}$ if $r=1, \ldots, n$, 
and $u_{t}$ if $r=n+1$. The same convention is used for the index $s$. The sum over repeated indices $r$ and $s$ is extended from 1 to $n+1$. As usual we denote $D u=\left(u_{1}, \ldots, u_{n}\right), \nabla u=\left(u_{1}, \ldots, u_{n}, u_{t}\right)$.

Now we state three lemmas for later use.

LEMMA 2.1. If $\left[a^{i j}\right]$ is an $n \times n$ symmetric positive definite matrix and $\left[b^{r s}\right]$ is an $(n+1) \times(n+1)$ negative semidefinite matrix then, for any $(n+1) \times n$ matrix $\left[\xi^{r i}\right]$ we have

$$
\xi^{r i} a^{i j} \xi^{s j} b^{r s} \leq 0
$$

PROOF. The result is well-known. It can be proved easily be diagonalizing the matrix $\left[a^{i j}\right]$.

LEMMA 2.2. Let $\left[a^{i j}\right]$ be an $n \times n$ symmetric positive definite matrix, and let $u$ be a $C^{2}(Q)$ function. If $q^{2}=u_{i} u_{i}$, then

$$
a^{i j} u_{k i} u_{k j} \geq a^{i j} q_{i} q_{j}
$$

Furthermore, if $\rho^{2}=u_{r} u_{r}$ then

$$
a^{i j} u_{r i} u_{r j} \geq a^{i j} \rho_{i} \rho_{j} .
$$

PROOF. Let us recall that the sum with respect to $i, j$ and $k$ is from 1 to $n$, whereas, the sum with respect to $r$ is from 1 to $n+1$ (consequently, $\rho^{2}=$ $q^{2}+u_{t} u_{t}$ ). This lemma is also well-known. For completeness let us prove (2.2) (the proof of (2.1) being similar). Let $A$ denote the matrix $\left[a^{i j}\right]$, let $H$ be the $(n+1) \times n$ Hessian matrix $\left[u_{r i}\right]$ and let $H^{T}$ be the transposed matrix of $H$. Since $A$ is symmetric and positive definite, the matrix $H A H^{T}$ is symmetric and positive semidefinite, and its trace is the left hand side of (2.2). The right hand side of (2.2) can be written as $a^{i j} u_{r i} u_{s j}\left(u_{r} / \rho\right)\left(u_{s} / \rho\right)$; but this is the quadratic form associated to $H A H^{T}$ and computed at the unit vector $\nabla u / \rho$. Hence (2.2) is true.

LEMMA 2.3. Let $\left[a^{i j}\right]$ be an $n \times n$ positive semidefinite matrix. Then the $2 n \times 2 n$ matrix

$$
B=\left|\begin{array}{ll}
{\left[a^{i j}\right]} & {\left[a^{i j}\right]} \\
{\left[a^{i j}\right]} & {\left[a^{i j}\right]}
\end{array}\right|
$$

is positive semidefinite. 
PROOF. For a given vector $\zeta \in \mathbb{R}^{2 n}$ let $\xi$ be the vector of $\mathbb{R}^{n}$ defined by the first $n$ coordinates of $\zeta$, and let $\eta$ be the vector defined by the last $n$ coordinates of $\zeta$. Then, the quadratic form $(B \zeta, \zeta)$ can be written as

$$
(B \zeta, \zeta)=a^{i j} \xi_{i} \xi_{j}+a^{i j} \xi_{i} \eta_{j}+a^{i j} \eta_{i} \xi_{j}+a^{i j} \eta_{i} \eta_{j}=a^{i j}\left(\xi_{i}+\eta_{i}\right)\left(\xi_{j}+\eta_{j}\right) .
$$

The lemma follows.

\section{Maximum principles involving the gradient}

THEOREM 3.1. Let $u(x, t)$ be a smooth solution of the parabolic equation

$$
F\left(u, D u, D^{2} u\right)=u_{t}
$$

in $Q$, where $F$ is a smooth function, $D u$ is the vector of the spatial derivatives $\left[u_{i}\right]$ and $D^{2} u=\left[u_{i j}\right]$. If $a^{i j}$ denote the partial derivatives of $F$ with respect to $u_{i j}$ we assume that $a^{i j}=a^{j i}$ and that for every $\xi \in \mathbb{R}^{n}$ and some $v>0$ the condition.

$$
a^{i j} \xi_{i} \xi_{j} \geq \nu \xi_{i} \xi_{i}
$$

holds. If $g(\tau)$ is a smooth positive decreasing function and $\rho^{2}=u_{r} u_{r}$ we define $w(x, t)$ by

$$
g(w) \rho=G\left(u_{1}, \ldots, u_{n}, u_{t}\right),
$$

where $G\left(\eta_{1}, \ldots, \eta_{n+1}\right)$ is a smooth concave function satisfying

$$
\eta_{r} \partial G / \partial \eta_{r}=G
$$

If $G\left(u_{1}, \ldots, u_{n}, u_{t}\right)$ and $\rho$ are positive corresponding to the solution $u(x, t)$ then the function $w(x, t)$ defined by (3.3), takes its maximum value on the parabolic boundary of $Q$, that is on $\Omega \times[0] \cup \partial \Omega \times[0, L]$.

PROOF. Recall that we sum from 1 to $n$ with respect to the repeated indices $i, j, k$ and from 1 to $n+1$ with respect to $r, s$. By (3.3) we derive

$$
\begin{aligned}
& \dot{g} \rho w_{i}+g \rho_{i}=\partial G / \partial \eta_{r} u_{r i}, \\
& \dot{g} \rho w_{t}+g \rho_{t}=\partial G / \partial \eta_{r} u_{r t},
\end{aligned}
$$

where $\dot{g}$ is the derivative of $g$. By (3.5) we obtain

$$
\dot{g} \rho w_{i j}+(\dot{g} \rho)_{j} w_{i}+\dot{g} \rho_{i} w_{j}+g \rho_{i j}=b^{r s} u_{r i} u_{s j}+\partial G / \partial \eta_{r} u_{r i j},
$$


where $\left[b^{r s}\right]$ is the $(n+1) \times(n+1)$ Hessian matrix of $G$. Since $G$ is concave, the matrix $\left[b^{r s}\right]$ is negative semidefinite. By (3.2) the $n \times n$ matrix $\left[a^{i j}\right]$ is positive definite; hence by Lemma 2.1 we have $a^{i j} b^{r s} u_{r i} u_{s j} \leq 0$ and (3.7) implies

$$
\dot{g} \rho a^{i j} w_{i j}+b^{i} w_{i}+g a^{i j} \rho_{i j} \leq \partial G / \partial \eta_{r} a^{i j} u_{r i j},
$$

where $b^{i}=a^{i j}\left((\dot{g} \rho)_{j}+\dot{g} \rho_{j}\right)$.

Since $\rho^{2}=u_{r} u_{r}$ we have

$$
\begin{aligned}
& \rho \rho_{i}=u_{r} u_{r i}, \quad \rho \rho_{t}=u_{r} u_{r t}, \\
& \rho \rho_{i j}=u_{r i} u_{r j}-\rho_{i} \rho_{j}+u_{r} u_{r i j} .
\end{aligned}
$$

By (2.2) $a^{i j} u_{r i} u_{r j}-a^{i j} \rho_{i} \rho_{j} \geq 0$. Hence (3.10) implies

$$
\rho a^{i j} \rho_{i j} \geq u_{r} a^{i j} u_{r i j}
$$

This inequality and (3.8) imply

$$
\dot{g} \rho a^{i j} w_{i j}+b^{i} w_{i} \leq\left(\partial G / \partial \eta_{r}-u_{r} g / \rho\right) a^{i j} u_{r i j} .
$$

By equation (3.1) we derive, for $r=1, \ldots, n+1$,

$$
a^{i j} u_{i j r}=u_{t r}-F_{u} u_{r}-u_{i r} \partial F / \partial u_{i} .
$$

By inserting the last equation into (3.12) we obtain

$$
-\dot{g} \rho a^{i j} w_{i j}-b^{i} w_{i} \geq g \rho_{t}-g \rho F_{u}-g \rho_{i} \frac{\partial F}{\partial u_{i}}-u_{t r} \frac{\partial G}{\partial \eta_{r}}+G F_{u}+u_{i r} \frac{\partial F}{\partial u_{i}} \frac{\partial G}{\partial \eta_{r}}
$$

where (3.9) and (3.4) have been used. Finally, (3.3), (3.5), (3.6) and the last inequality lead to

$$
a^{i j} w_{i j}+d^{i} w_{i} \geq w_{t}
$$

where $d^{i}=b^{i} /(\dot{g} \rho)+\partial F / \partial u_{i}$. Now the theorem follows by the well-known maximum principle for parabolic inequalities [11, p. 173].

REMARK 3.1. If $F_{u}=0$ in equation (3.1), then condition (3.4) can be omitted in Theorem 3.1. 
THEOREM 3.2. Let $u(x, t)$ be a smooth solution of the equation

$$
\Delta u+2 h\left(q^{2}\right) u_{i} u_{j} u_{i j}=u_{t}
$$

in $Q=\Omega \times(0, L]$, where $\Delta$ is the Laplace operator, $q^{2}=u_{i} u_{i}$ and $h(\tau)$ is a smooth function satisfying $1+2 q^{2} h\left(q^{2}\right)>0$. We define $T\left(q^{2}\right)=1+2 q^{2} h\left(q^{2}\right)$ and assume there is a finite $\gamma$ such that

$$
1+q^{2} \dot{T} / T \leq \gamma,
$$

where $\dot{T}$ is the derivative of $T$ with respect to $q^{2}$. If $f(\tau)$ is a smooth positive function satisfying

$$
\left(\dot{f} f^{-2 \gamma}\right)^{\cdot} \geq 0
$$

then the function

$$
\Phi(x, t)=q f(u)
$$

takes its maximum value on $\Omega \times[0] \cup \partial \Omega \times[0, L]$.

ProOF. By (3.17) we derive:

$$
\begin{gathered}
\Phi_{i}=q_{i} f+q \dot{f} u_{i}, \quad \Phi_{t}=q_{t} f+q \dot{f} u_{t}, \\
\Phi_{i j}=q_{i j} f+q_{i} \dot{f} u_{j}+q_{j} \dot{f} u_{i}+q \ddot{f} u_{i} u_{j}+q \dot{f} u_{i j} .
\end{gathered}
$$

We set $a^{i j}=\delta^{i j}+2 h\left(q^{2}\right) u_{i} u_{j}$, where $\delta^{i j}$ is the Kronecker delta. The matrix $\left[a^{i j}\right]$ is symmetric and its eigenvalues are 1 and $1+2 q^{2} h\left(q^{2}\right)$. Hence it is positive definite by virtue of the assumption $1+2 q^{2} h\left(q^{2}\right)>0$. With this notation, equation (3.14) reads $a^{i j} u_{i j}=u_{t}$, and (3.19) implies

$$
a^{i j} \Phi_{i j}=f a^{i j} q_{i j}+2 \dot{f} a^{i j} q_{i} u_{j}+q \ddot{f} a^{i j} u_{i} u_{j}+q \dot{f} u_{t} .
$$

By the definition of $q$ we derive

$$
q q_{i}=u_{k} u_{k i}, \quad q q_{t}=u_{k} u_{k t}
$$

and

$$
q q_{i j}=u_{k i} u_{k j}, q_{i} q_{j}+u_{k} u_{k i j}
$$

The last equation and inequality (2.1) of Lemma 2.2 imply

$$
q a^{i j} q_{i j} \geq u_{k} a^{i j} u_{k i j}
$$


By the equation $a^{i j} u_{i j}=u_{t}$ we derive

$$
u_{k} a^{i j} u_{i j k}=u_{k} u_{t k}-u_{k} a_{k}^{i j} u_{i j}=u_{k} u_{t k}-2 u_{k}\left(2 \dot{h} q q_{k} u_{i} u_{j}+h u_{i k} u_{j}+h u_{i} u_{j k}\right) u_{i j} \text {. }
$$

The latter and equations (3.21) give

$$
u_{k} a^{i j} u_{i j k}=q q_{t}-2 q^{2}\left(2 \dot{h}\left(q_{k} u_{k}\right)^{2}+2 h q_{j} q_{j}\right) .
$$

By using (3.23), (3.22) and the last of equations (3.18), (3.20) gives

$$
a^{i j} \Phi_{i j} \geq \Phi_{t}-2 f q\left(2 \dot{h}\left(q_{i} u_{i}\right)^{2}+2 h q_{i} q_{i}\right)+2 \dot{f} a^{i j} q_{i} u_{j}+q \ddot{f} a^{i j} u_{i} u_{j} .
$$

By (3.18) it follows that

$$
\begin{aligned}
q_{i} u_{i} & =f^{-1} \Phi_{i} u_{i}-q^{3} f^{-1} \dot{f}, \\
q_{i} q_{i} & =f^{-1} \Phi_{i} q_{i}-q f^{-1} \dot{f} q_{i} u_{i} \\
& =f^{-1} \Phi_{i} q_{i}-q f^{-2} \dot{f} \Phi_{i} u_{i}+q^{4} f^{-2} \dot{f}^{2}, \\
a^{i j} q_{i} u_{j} & =a^{i j}\left(f^{-1} \Phi_{i}-f^{-1} \dot{f} q u_{i}\right) u_{j} \\
& =f^{-1} a^{i j} \Phi_{i} u_{j}-f^{-1} \dot{f} q a^{i j} u_{i} u_{j} .
\end{aligned}
$$

Insertion of (3.25), (3.26) and (3.27) into (3.24) leads to

$$
a^{i j} \Phi_{i j}+b^{i} \Phi_{i} \geq \Phi_{t}-2 f^{-1} \dot{f}^{2} q^{5}\left(2 \dot{h} q^{2}+2 h\right)-2 f^{-1} \dot{f}^{2} q a^{i j} u_{i} u_{j}+q \ddot{f} a^{i j} u_{i} u_{j},
$$

where $b^{i}$ are expressions depending on $f, h, a^{i j}, u_{i}, q_{i}$ and $\Phi_{i}$ (but not on $\Phi_{t}$ ).

Since $2 \dot{h} q^{2}+2 h=\dot{T}$ and $a^{i j} u_{i} u_{j}=q^{2} T,(3.28)$ can be rewritten as

$$
a^{i j} \Phi_{i j}+b^{i} \Phi_{i} \geq \Phi_{t}+q^{3} T\left(\ddot{f}-2 f^{-1} \dot{f}^{2}\left(1+q^{2} \dot{T} / T\right)\right) .
$$

Since, by assumption, $1+q^{2} \dot{T} / T \leq \gamma$, inequality (3.29) implies

$$
a^{i j} \Phi_{i j}+b^{i} \Phi_{i}-\Phi_{t} \geq q^{3} T\left(\ddot{f}-2 \gamma f^{-1} \dot{f}^{2}\right)=q^{3} T f^{2 \gamma}\left(\dot{f} f^{-2 \gamma}\right) \cdot \geq 0,
$$

where assumption (3.16) has been used in the last step. The theorem follows by the maximum principle for parabolic equations.

THEOREM 3.3. Under the same notation and assumptions of Theorem 3.2, if $h \leq 0$ and $\gamma=1$, then the function

$$
\Phi(x, t)=q f(v)
$$

attains its maximum value on $\Omega \times[0] \cup \partial \Omega \times[0, L]$. Here $q$ and $f$ are the same as in Theorem 3.2, whereas $v$ is any solution of the equation.

$$
\Delta v+2 h\left(q^{2}\right) u_{i} u_{j} v_{i j}=v_{t}
$$


PROOF. Arguing as in the proof of Theorem 3.2 we obtain

$$
a^{i j} \Phi_{i j} \geq \Phi_{t}-2 f q\left[2 \dot{h}\left(q_{i} u_{i}\right)^{2}+2 h q_{i} q_{i}\right]+2 \dot{f} a^{i j} q_{i} v_{j}+q \ddot{f} a^{i j} v_{i} v_{j}
$$

Our assumption $\gamma=1$ implies $2 \dot{h} q^{2}+2 h=\dot{T} \leq 0$. This inequality, together with the Schwarz inequality $\left(q_{i} u_{i}\right)^{2} \leq q_{i} q_{i} u_{k} u_{k}$ and the assumption $h \leq 0$, makes the quantity between square brackets in (3.32) non-positive, hence it can be deleted.

On the other side, by (3.30) we derive

$$
q_{i}=f^{-1} \Phi_{i}-q f^{-1} \dot{f} v_{i}
$$

hence (3.32) implies

$$
a^{i j} \Phi_{i j}+b^{i} \Phi_{i}-\Phi_{t} \geq q a^{i j} v_{i} v_{j}\left(\ddot{f}-2 f^{-1} \dot{f}^{2}\right)=q a^{i j} v_{i} v_{j} f^{2}\left(\dot{f} f^{-2}\right) \cdot \geq 0,
$$

where $b^{i}=-2 f^{-1} \dot{f} a^{i j} v_{j}$ and condition (3.15) (with $\gamma=1$ ) has been used in the last step. The theorem follows.

COROLlaRY 3.1. Let $u$ satisfy $u_{t}=\Delta u$ in $Q=\Omega \times[0, L]$ with $q(x, t)=0$ on $\partial \Omega \times[0, L]$, and let $v$ be a positive solution of $v_{t}=\Delta v$ in $Q$ with $v(x, t)=1$ on $\Omega \times[0]$. Then we have

$$
q(x, t) \leq q(\bar{x}, 0) v(x, t)
$$

for all $(x, t) \in Q$ and some $\bar{x} \in \Omega$.

PROOF. The function $f(v)=v^{-1}$ satisfies (3.16) with $\gamma=1$, hence, by Theorem 3.3, the expression $q v^{-1}$ attains its maximum value on $\Omega \times[0] \cup \partial \Omega \times$ $[0, L]$. But $q=0$ on $\partial \Omega \times[0, L]$ and $v=1$ on $\Omega \times[0]$ by assumption. The corollary follows.

\section{A maximum principle for the concavity function}

If $\alpha$ and $\beta$ are positive real numbers satisfying $\alpha+\beta=1$, if $\lambda$ is a real number, and if $u(x, t)$ is a function defined in $Q$, let us define

$$
\begin{aligned}
\Phi(x, y, t, \tau) & =e^{\lambda \theta}[u(z, \theta)-\alpha u(x, t)-\beta u(y, \tau)], \\
z & =\alpha x+\beta y, \\
\theta & =\alpha t+\beta \tau,
\end{aligned}
$$

for all $x, y$ such that $x, y, z \in \Omega$ and for all $t, \tau \in(0, L]$. 
THEOREM 4.1. Let $u(x, t)$ be a smooth function defined in $Q$ and satisfying equation (1.2). Suppose that the $n \times n$ matrix $\left[a^{i j}(D u)\right]$ of (1.2) is positive semidefinite for any vector $D u$, that the function $b(x, t, u, D u)$ is jointly concave with respect to $(x, t, u)$, and that the derivative $\partial b / \partial u$ is bounded from below. Then the function $\phi$ defined in (4.1) with $\lambda$ satisfying

$$
\partial b / \partial u-\lambda>0
$$

has no positive maximum for $x, y, z \in \Omega$ and $t, \tau \in(0, L]$.

Proof. Assume to the contrary that $(x, y, t, \tau)$ is a positive maximum for $\Phi$ with $x, y, z \in \Omega$ and $t, \tau \in(0, L]$. At this point we have

$$
\begin{aligned}
& \Phi_{x_{i}}=\alpha e^{\lambda \theta}\left[u_{z_{i}}(z, \theta)-u_{x_{i}}(x, t)\right]=0, \\
& \Phi_{y_{i}}=\beta e^{\lambda \theta}\left[u_{z_{i}}(z, \theta)-u_{y_{i}}(y, \tau)\right]=0 .
\end{aligned}
$$

Equations (4.3) - (4.4) imply that the spatial gradient $D u$ is the same at the three points $(z, \theta),(x, t),(y, \tau)$. We also have

$$
\begin{aligned}
& \Phi_{t}=\lambda \alpha \Phi+e^{\lambda \theta}\left[\alpha u_{\theta}(z, \theta)-\alpha u_{t}(x, t)\right] \geq 0, \\
& \Phi_{\tau}=\lambda \beta \Phi+e^{\lambda \theta}\left[\beta u_{\theta}(z, \theta)-\beta u_{\tau}(y, \tau)\right] \geq 0,
\end{aligned}
$$

from which it follows that

$$
\lambda \Phi+e^{\lambda \theta}\left[u_{\theta}(z, \theta)-\alpha u_{t}(x, t)-\beta u_{\tau}(y, \tau)\right] \geq 0 .
$$

At the maximum point $(x, y, t, \tau)$, the Hessian matrix

$$
H=\left|\begin{array}{ll}
{\left[\Phi_{x_{i} x_{j}}\right]} & {\left[\Phi_{x_{i} y_{j}}\right]} \\
{\left[\Phi_{y_{i} x_{j}}\right]} & {\left[\Phi_{y_{i} y_{j}}\right]}
\end{array}\right|
$$

is negative semidefinite. By Lemma 2.3, the matrix $B$ defined in (2.3) by using the matrix $\left[a^{i j}(D u)\right]$ of the equation (1.2) (computed at any of the points $(z, \theta)$, $(x, t),(y, \tau))$ is positive semidefinite. Consequently, the matrix $B H$ is negative semidefinite and its trace is non positive. Hence

$$
0 \geq a^{i j} \Phi_{x_{i} x_{j}}+a^{i j} \Phi_{x_{i} y_{j}}+a^{i j} \Phi_{y_{i} x_{j}}+a^{i j} \Phi_{y_{i} y_{j}} .
$$

By (4.1) we derive

$$
\begin{aligned}
& \Phi_{x_{i} x_{j}}=e^{\lambda \theta}\left[\alpha^{2} u_{z_{i} z_{j}}(z, \theta)-\alpha u_{x_{i} x_{j}}(x, t)\right], \\
& \Phi_{x_{i} y_{j}}=\Phi_{y_{i} x_{j}}=e^{\lambda \theta} \alpha \beta u_{z_{i} z_{j}}(z, \theta), \\
& \Phi_{y_{i} y_{j}}=e^{\lambda \theta}\left[\beta^{2} u_{z_{i} z_{j}}(z, \theta)-\beta u_{y_{i} y_{j}}(y, \tau)\right] .
\end{aligned}
$$


Insertion of the last equations into (4.6) leads to

$$
0 \geq e^{\lambda \theta}\left[(\alpha+\beta)^{2} a^{i j} u_{z_{i} z_{j}}(z, \theta)-\alpha a^{i j} u_{x_{i} x_{j}}(x, t)-\beta a^{i j} u_{y_{i} y_{j}}(y, \tau)\right] .
$$

The latter inequality and the equation (1.2) give

$$
\begin{array}{r}
0 \geq e^{\lambda \theta}\left[b(z, \theta, u)+u_{\theta}(z, \theta)-\alpha b(x, t, u)-\alpha u_{t}(x, t)\right. \\
\left.-\beta b(y, \tau, u)-\beta u_{\tau}(y, \tau)\right]
\end{array}
$$

where $D u(z, \theta)=D u(x, t)=D u(y, \tau)$ are suppressed in the expression of $b$. Using the concavity of $b$ and inequality (4.5), (4.7) implies

$$
\begin{gathered}
0 \geq e^{\lambda \theta}[b(z, \theta, u(z, \theta))-b(z, \theta, \alpha u(x, t)+\beta u(y, \tau))]-\lambda \Phi \\
=(\partial b / \partial u-\lambda) \Phi .
\end{gathered}
$$

Since, by assumption, $\partial b / \partial u-\lambda>0$ and the value of $\Phi$ at the point of maximum $(x, y, t, \tau)$ is positive, $(4.8)$ is a contradiction. The theorem is proved.

COROLLARY 4.1. With the notation and assumptions of Theorem 4.1, if the concavity function $C(x, y, t, \tau)$ defined in (1.3) is non-positive when $x, y, z, \in$ $\Omega$, and one of $t, \tau=0$, and when $t, \tau \in(0, L]$, and one of $x, y, z \in \partial \Omega$, then $C(x, y, t, \tau) \leq 0$ for all $x, y$ in $\Omega$ and $t, \tau$ in $(0, L]$.

Proof. Since the inequality $C(x, y, t, \tau) \leq 0$ implies $\Phi(x, y, t, \tau) \leq 0$ and vice versa, the corollary follows by Theorem 4.1 .

\section{References}

[1] A. Buttu, 'An angle's maximum principle for the gradient of solutions of parabolic equations', Boll. Un. Mat. Ital. A 2 (1988), 405-408.

[2] A. Haraux, Nonlinear evolution equations-global behaviour of solutions, Lecture Notes in Math. 841 (Springer, Berlin, 1981).

[3] D. Henry, Geometric theory of semilinear parabolic equations, Lecture Notes in Math. 840 (Springer, Berlin, 1981).

[4] B. Kawohl, Rearrangements and convexity of level sets in PDE, Lecture Notes in Math. 1150 (Springer, Berlin, 1985).

[5] N. Korevaar, 'Capillarity surface convexity above convex domains', Indiana Univ. Math. J. 32 (1983), 73-82. 
[6] - 'Convex solutions to nonlinear elliptic and parabolic boundary value problems', Indiana Univ. Math. J. 32 (1983), 603-614.

[7] L. P. Payne and G. A. Philippin, 'On some maximum principles involving harmonic functions and their derivatives', SIAM J. Math. Anal. 10 (1979), 96-104.

[8] G. A. Philippin, 'On a free boundary problem in electrostatic', Math. Methods Appl. Sci. 12 (1990), 387-392.

[9] G. A. Philippin and L. P. Payne, 'On the conformal capacity problem', Sympos. Math. 30 (1989), 119-136.

[10] G. Porru and F. Ragnedda, 'Convexity properties for solutions of some second order elliptic semilinear equations', Appl. Anal. 37 (1990), 1-18.

[11] M. H. Protter and H. F. Weinberger, Maximum principles in differential equations (PrenticeHall, Englewood Ciffs, 1967).

[12] C. Pucci, 'An angle's maximum principle for the gradient of solutions of elliptic equations', Boll. Un. Mat. Ital. A 1 (1987), 135-139.

[13] —_. 'A maximum principle related to level surfaces of solutions of parabolic equations', J. Austral. Math. Soc. (Series A) 46 (1989), 1-7.

Dipartimento di Matematica

Universita degli studi di Cagliari

Via Ospedale 72

09124 Cagliari

Italy
Dipartimento di Matematica Universita degli studi di Cagliari

Via Ospedale 72 09124 Cagliari 